\title{
Sensitivity analysis of the meteorological preprocessor MPP-FMI 3.0 using algorithmic differentiation
}

\author{
John Backman ${ }^{1}$, Curtis R. Wood ${ }^{1}$, Mikko Auvinen ${ }^{1,2}$, Leena Kangas ${ }^{1}$, Hanna Hannuniemi ${ }^{1}$, Ari Karppinen ${ }^{1}$, and \\ Jaakko Kukkonen ${ }^{1}$ \\ ${ }^{1}$ Atmospheric Composition Research, Finnish Meteorological Institute, Helsinki, Finland \\ ${ }^{2}$ Department of Physics, Division of Atmospheric Sciences, University of Helsinki, Helsinki, Finland \\ Correspondence to: John Backman (john.backman@fmi.fi)
}

Received: 16 December 2016 - Discussion started: 13 January 2017

Revised: 30 August 2017 - Accepted: 3 September 2017 - Published: 17 October 2017

\begin{abstract}
The meteorological input parameters for urbanand local-scale dispersion models can be evaluated by preprocessing meteorological observations, using a boundarylayer parameterisation model. This study presents a sensitivity analysis of a meteorological preprocessor model (MPPFMI) that utilises readily available meteorological data as input. The sensitivity of the preprocessor to meteorological input was analysed using algorithmic differentiation (AD). The AD tool used was TAPENADE. The AD method numerically evaluates the partial derivatives of functions that are implemented in a computer program. In this study, we focus on the evaluation of vertical fluxes in the atmosphere and in particular on the sensitivity of the predicted inverse Obukhov length and friction velocity on the model input parameters. The study shows that the estimated inverse Obukhov length and friction velocity are most sensitive to wind speed and second most sensitive to solar irradiation. The dependency on wind speed is most pronounced at low wind speeds. The presented results have implications for improving the meteorological preprocessing models. AD is shown to be an efficient tool for studying the ranges of sensitivities of the predicted parameters on the model input values quantitatively. A wider use of such advanced sensitivity analysis methods could potentially be very useful in analysing and improving the models used in atmospheric sciences.
\end{abstract}

\section{Introduction}

Any urban- or local-scale dispersion model requires specific information about the state of the atmospheric boundary layer (ABL) as input values. This information can be estimated from available meteorological observations by socalled meteorological preprocessors (e.g. Van Ulden and Holtslag, 1985). This allows for the use of advanced meteorological input data into the models, even when no atmospheric turbulence measurements would be available. These evaluations are commonly done by applying an energy-flux method that estimates turbulent heat and momentum fluxes in the boundary layer to derive desired boundary-layer scaling parameters (e.g. Fisher et al., 2001; Van Ulden and Holtslag, 1985)

The urban-scale dispersion models at the Finnish Meteorological Institute (FMI) rely on advanced meteorological input from a meteorological preprocessor that is mainly based on the boundary-layer parameterisation of Van Ulden and Holtslag (1985). These dispersion models include, e.g. a Gaussian road network dispersion model (CAR-FMI, Kukkonen et al., 2001; Kauhaniemi et al., 2008) and an urban multiple source Gaussian dispersion model (UDM-FMI; Karppinen et al., 2000b). The models are used to model emissions, dispersion, and transformation of pollution for urban-scale areas. The present work focuses on the meteorological preprocessor model and its sensitivity to model input, whereas dispersion models (not discussed here) motivate the study.

Model sensitivity studies can be done with precision using algorithmic differentiation (AD), which is a technique to compute accurate partial derivatives of functions that are implemented by computer programmes. In the context of 
$\mathrm{AD}$, a computer program is viewed as a complex function that is composed of a sequence of basic mathematical operations. $\mathrm{AD}$ is a systematic technique to apply the chain rule of differentiation to this sequence of numerical operations in a manner that does not involve inaccuracies (Griewank and Walther, 2008). In this study, a source-transformation AD tool called TAPENADE (Hascoet and Pascual, 2013) is employed to differentiate the procedures of a meteorological preprocessor. TAPENADE was chosen because it is an easy-to-use Fortran source-transformation tool that is free for academic use, actively supported and developed, and is well documented.

Other source-transformation $\mathrm{AD}$ tools for Fortran are also available (e.g. OpenAD) and a representative list can be found from the community-driven portal for algorithmic differentiation (http://www.autodiff.org). A sourcetransformation tool approaches the differentiation by analysing the source code of a given computer program and generating an augmented source code which contains, in addition to the original operations, instructions that carry out their chain rule differentiated versions. As these differentiated statements accompany each relevant mathematical operation in the source code, they propagate the derivative information across the entire program, providing exact sensitivity information (to machine precision) on how the inputs of the program influence its results. In this way, a standard (Fortran in this case) compiler can be used, which is not the case for the other AD methods (such as operator overloading and $\mathrm{AD}$-enabled compilers).

AD has applications that span multiple disciplines of science such as engineering, physics, chemistry, and medicine, where it can be used for sensitivity analyses, optimisation, and inverse problem solving, etc. (Griewank and Walther, 2008). In fact, $A D$ has applications wherever partial derivatives of computer programmes can be made useful. It is not the intention to give a full literature review of research that has benefited from AD but rather a brief overview of its applications in geophysical research and in particular using TAPENADE.

The AD tool TAPENADE has been used for a variety of different physics models as follows. A general purpose atmospheric radiative transfer model for remote sensing applications made use of the superior numerical accuracy of $\mathrm{AD}$, in comparison to finite difference perturbations, for evaluation of satellite trace gas spectra (Schreier et al., 2014). Moreover, the AD method was later recommended for the same model due to lower computational cost and greater numerical accuracy when solving non-linear inverse radiative transfer problem through iteration (Schreier et al., 2015). A meteorologychemistry coupled model also made use of AD source transformation when developing a four-dimensional variational data assimilation procedure for the model (Guerrette and Henze, 2015). TAPENADE has also been used for a sensitivity study of a sea-ice model to determine optimal model parameters in a minimisation algorithm (Kim et al., 2006).
More information and literature on $\mathrm{AD}$ can be found at www.autodiff.org.

The sensitivity on input data of the above-mentioned meteorological preprocessing method has not previously been systematically investigated. The aim of this study is to quantitatively determine the sensitivities of meteorological output parameters on model input for the meteorological preprocessor MPP-FMI (Karppinen et al., 1997, 2000a). This procedure is useful for analysing in detail the functioning of the computer program corresponding to the model MPP-FMI. The modelled sensitivities can also be compared to what would be physically feasible, based on a consideration of the relevant atmospheric processes. This will provide a useful additional test regarding the correct functioning of the computer code and the numerical procedures of the MPP-FMI model. Such a thorough and quantitative sensitivity analysis also provides new information and insights regarding the further refinement of such models.

\section{Methods}

\subsection{The meteorological preprocessor MPP-FMI}

The meteorological preprocessor is used to estimate turbulent fluxes, atmospheric stability, and boundary-layer scaling parameters based on meteorological observations at fixed locations. The scope of this study is to determine the sensitivity of this model for deriving the vertical fluxes in the boundary layer. However, we have not addressed the routines within the MPP-FMI model that deal with the vertical temperature gradient and hence mixing height which are obtained from temperature profiles provided by radiosondes (Karppinen et al., 2001). Mixing height is another key parameter for the modelling of dispersion of pollutants because it determines the spread of pollutants particularly vertically, and so any future dispersion-model sensitivity study, based on the present work, would naturally also use mixing height as an input. The scope of the present study is depicted in Fig. 1.

The meteorological observations used by the MPP-FMI model as input comprise temperature $\left(T_{2}\right)$, wind speed $(U)$ and wind direction at a height of $10 \mathrm{~m}$, amount of predominant clouds $\left(C_{\mathrm{C}}\right)$, cloud height $\left(C_{\mathrm{Z}}\right)$, sunshine fraction, state of the ground (wet, dry, snow, ice, etc.), and precipitation. These are needed by the preprocessor in order to model boundary-layer scaling parameters required by urban-scale dispersion models.

MPP-FMI is originally based on the work by Van Ulden and Holtslag (1985) with modifications that make the parameterisation more suitable for high latitudes and urban areas (Karppinen et al., 1997, 2000a). Central to this method is the surface heat-budget equation:

$Q^{*}-Q_{\mathrm{G}}=Q_{\mathrm{H}}+Q_{\mathrm{E}}$. 


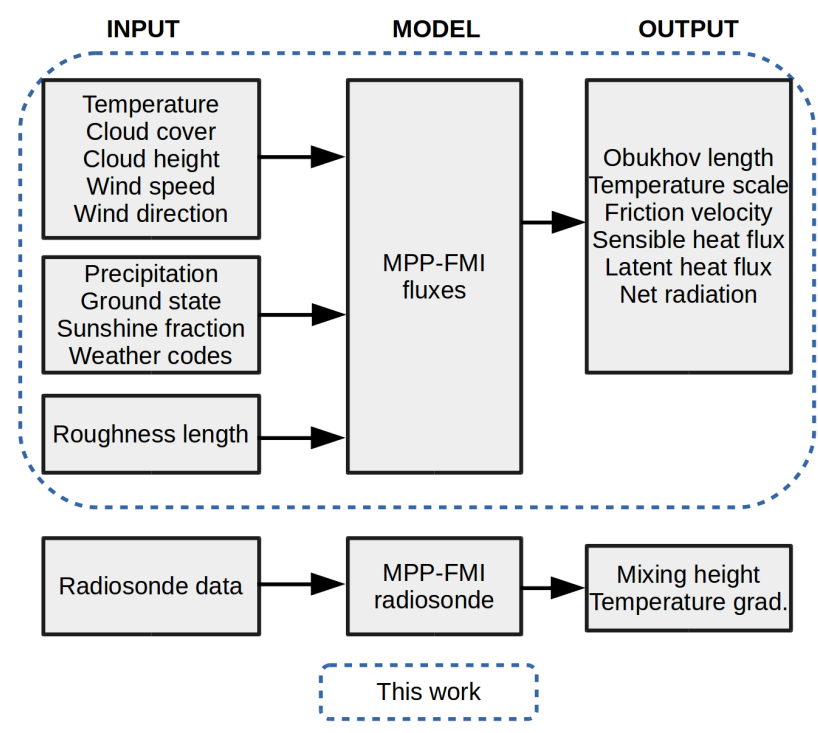

Figure 1. A schematic diagram on the flow of information of the meteorological preprocessor MPP-FMI.

In Eq. (1), $Q^{*}$ is the surface net radiation, $Q_{\mathrm{G}}$ is the soil heat flux, $Q_{\mathrm{H}}$ is the sensible heat flux, and $Q_{\mathrm{E}}$ is the latent heat flux. The terms that comprise Eq. (1) are not commonly available from measurements (although there are measurements of eddy covariance at some research sites; Wood et al., 2013) and are therefore estimated by the meteorological preprocessor. A comprehensive description of MPP-FMI is already available in the literature (Karppinen et al., 1997). However, a brief overview of the model structure will be presented in the following for convenience.

First, the meteorological preprocessor estimates available energy $Q^{*}-Q_{\mathrm{G}}$ by decomposing the terms into components of (i) net shortwave radiation using incoming shortwave radiation and albedo, (ii) net longwave radiation from surface radiative temperature and cloud-base radiation temperature (specific for MPP-FMI) using a constant dry adiabatic lapserate and cloud-base height, and (iii) estimated heat flux into the ground from estimated temperature difference between the ground and a reference height of $50 \mathrm{~m}$. Then, the term $Q_{\text {E }}$ is estimated using a simplified Penman-Monteith equation (Van Ulden and Holtslag, 1985). Consequently, an estimate of the sign of $Q_{\mathrm{H}}$ is obtained, which will determine if the subsequent calculations are to be done using stability functions for stable or unstable conditions.

According to surface-layer similarity theory, both friction velocity $\left(u_{*}\right)$ and temperature scale for turbulent heat transfer $\left(\theta_{*}\right)$ can be expressed as vertical profiles. For $u_{*}$, which is a measure of the surface production of turbulent kinetic energy, the equation is

$u_{*}=\frac{U(z) k}{\ln \left(\frac{z}{z_{0}}\right)-\psi_{\mathrm{M}}\left(\frac{z}{L}\right)+\psi_{\mathrm{M}}\left(\frac{z_{0}}{L}\right)}$.
In Eq. (2), $U$ is wind speed at height $z, z_{0}$ is the surface roughness length, $k$ is the von Karman constant, and the terms $\psi_{\mathrm{M}}$ are stability functions; see Appendix A for details. $L$ is the Obukhov length, which is an atmospheric stability measure that describes the relative importance of surface production of turbulence due to shear stress and buoyancy forces.

Similarly to $u_{*}, \theta_{*}$ can be written as

$\theta_{*}=\frac{k\left[\theta\left(z_{2}\right)-\theta\left(z_{1}\right)\right]}{\ln \left(\frac{z_{2}}{z_{1}}\right)-\psi_{\mathrm{H}}\left(\frac{z_{2}}{L}\right)+\psi_{\mathrm{H}}\left(\frac{z_{1}}{L}\right)}$,

where $z_{1}$ and $z_{2}$ are arbitrary heights in the surface layer, $\theta$ is the potential temperature at the respective heights, and the terms $\psi_{\mathrm{H}}$ are stability functions. Both Eqs. (2) and (3) and their respective stability functions are used as described in Van Ulden and Holtslag (1985). Using Eq. (3), $\theta\left(z_{2}\right)$ at a reference height of $50 \mathrm{~m}$ can be modelled from measurements of $\theta\left(z_{1}\right)$. This is done by solving $\theta_{*}$ from the definition of $L$,

$L=\frac{u_{*}^{2} \theta}{k g \theta_{*}}$,

and substituting it into Eq. (3). In Eq. (4), $g$ is the acceleration due to gravity. This completes the modelling of $\theta_{*}$ using surface-layer similarity theory using the profile method (Van Ulden and Holtslag, 1985).

In addition to Eqs. (3) and (4), $\theta_{*}$ can also be estimated using the energy-budget method derived from the modified Penman-Monteith equation:

$\theta_{*}=\left(\frac{\alpha S}{S+1}-1\right)\left(\frac{Q^{*}-G}{\rho c_{\mathrm{p}} u_{*}}\right)+\alpha \theta_{\mathrm{d}}$,

where $\alpha$ is the Priestley-Taylor moisture parameter, $S$ is the saturation enthalpy curve of water vapour, $\rho$ the density of air, $c_{\mathrm{p}}$ is the specific heat capacity of air, and $\theta_{\mathrm{d}}$ is an empirical temperature scale. The derivation of Eq. (5) is done using the equations in Van Ulden and Holtslag (1985). In MPPFMI, however, the parameterisation of $S$ is different from that of Van Ulden and Holtslag (1985) in order to extend the temperature range of the parameterisation. Both parameterisations are very similar and are solely functions of surface temperature.

Finally, the value for $L$ is found iteratively by changing $L$ until $\theta_{*}$ from the profile method is equal to $\theta_{*}$ from the energy-budget method of Eq. (5); namely, Eq. (5) is equal to $u_{*}^{2} \theta /(\mathrm{kgL})$. This iteration will consequently impact $u_{*}$ and $\theta_{*}$ as described above. In addition, $Q^{*}, G, Q_{\mathrm{H}}$, and $Q_{\mathrm{E}}$ will also change during the iteration because of the stability functions of Eqs. (2) and (3).

\subsection{Algorithmic differentiation}

Algorithmic differentiation (AD) deals with the numerical evaluation of derivatives of functions that are implemented 
in a computer programme. Any computer program, no matter how complex, performs a sequence of arithmetic operations (addition, subtraction, division, etc.) or elementary functions (exponential, trigonometric, etc.) whose derivatives are known. AD exploits this fact by applying the chain rule of differentiation to the entire sequence of operations within the program (Griewank and Walther, 2008). This systematic approach yields numerical derivative values at machine precision, which describe how the program's results (i.e. outputs) depend on its inputs. The AD method performs each differentiation operation at machine precision and does not employ approximate techniques, such as finite differences. For this reason, $\mathrm{AD}$ does not suffer from truncation or round-off errors. The evaluation of finite differences is further complicated if input variables differ by orders of magnitude. By choosing the AD method, the tedious and imprecise evaluation can be avoided.

$\mathrm{AD}$ is further separated into two modes: a forward mode or a reverse mode (Griewank and Walther, 2008). Here, the discussion will be limited to the forward mode, which has been employed in this study. As a starting point, consider an arbitrary computer program that takes $n$ input variables and returns $m$ outputs. It can be described as a vector-valued function:

$\boldsymbol{y}=F(\boldsymbol{x})$,

such that the function $F$ maps $\mathbb{R}^{n} \rightarrow \mathbb{R}^{m}$ where $\boldsymbol{x} \in \mathbb{R}^{n}$ defines the input and $\boldsymbol{y} \in \mathbb{R}^{m}$ the output vectors.

Application of the forward-mode AD to Eq. (6) yields a new implementation of the program, which, in addition to the original function evaluation, evaluates its differential:

$\dot{\boldsymbol{y}}_{k}=F^{\prime}(\boldsymbol{x}) \dot{\boldsymbol{x}}_{k}$.

In Eq. (7), $F^{\prime}(x) \in \mathbb{R}^{m \times n}$ defines the Jacobian matrix, which contains all first-order partial derivatives $\partial y / \partial x$, and $\dot{\boldsymbol{x}}_{k}=$ $\left(\partial x_{1} / \partial x_{k}, \ldots, \partial x_{k} / \partial x_{k}, \ldots, \partial x_{n} / \partial x_{k}\right)^{T}$ is the seeding vector, which can be viewed as the $k$ th unit vector that operates on the Jacobian. The result is the $k$ th column from the Jacobian matrix $\dot{\boldsymbol{y}}_{k}=\left(\partial y_{1} / \partial x_{k}, \partial y_{2} / \partial x_{k}, \ldots, \partial y_{m} / \partial x_{k}\right)^{T}$ which yields the dependency of all outputs with respect to the userspecified $x_{k}$ input parameter. In the forward-mode differentiated computer program, the derivative evaluations based on the chain rule contained in Eq. (7) are performed following the same order as the associated operations in Eq. (6), but always such that the derivative operations are executed before their corresponding step in the original program have completed.

A typical goal in sensitivity analysis is to obtain the full Jacobian. Utilising forward-mode $\mathrm{AD}$, this is achieved by repeating the computation of Eq. (7) $n$ times to yield all the columns of the Jacobian matrix. This is best illustrated with an example matrix (Eq. 8) where the first column of the Jacobian is chosen. Thus, for a given input $\boldsymbol{x}$, one can construct the Jacobian using $\mathrm{AD}$ and extract the derivatives of the output of interest at that point. This procedure can then be repeated for any number of points.

$$
\dot{\boldsymbol{y}}_{1}=\underbrace{\left[\begin{array}{c}
\frac{\partial y_{1}}{\partial x_{1}} \\
\frac{\partial y_{2}}{\partial x_{1}} \\
\vdots \\
\frac{\partial y_{m}}{\partial x_{1}}
\end{array}\right]}_{\dot{\boldsymbol{y}}_{k=1} \in \mathbb{R}^{m}}=\underbrace{\left[\begin{array}{cccc}
\frac{\partial y_{1}}{\partial x_{1}} & \frac{\partial y_{1}}{\partial x_{2}} & \cdots & \frac{\partial y_{1}}{\partial x_{n}} \\
\frac{\partial y_{2}}{\partial x_{1}} & \frac{\partial y_{2}}{\partial x_{2}} & \cdots & \frac{\partial y_{2}}{\partial x_{n}} \\
\vdots & \vdots & \ddots & \vdots \\
\frac{\partial y_{m}}{\partial x_{1}} & \cdots & \cdots & \frac{\partial y_{m}}{\partial x_{n}}
\end{array}\right]}_{F^{\prime}(\boldsymbol{x}) \in \mathbb{R}^{m \times n}} \underbrace{\left[\begin{array}{c}
1 \\
0 \\
\vdots \\
0
\end{array}\right]}_{\dot{\boldsymbol{x}}_{k=1} \in \mathbb{R}^{m \times n}}
$$

The reverse mode of AD is not applied in this work because the number of input variables is roughly the same as the number of output variables $(m \approx n)$. The reverse mode should be favoured when $n \gg m$ because the reverse mode constructs the Jacobian one row at a time and is therefore more efficient (Griewank and Walther, 2008). Again, the differentiation was performed using the AD tool called TAPENADE (Hascoet and Pascual, 2013). TAPENADE has been developed by the French National Institute for computer science and applied mathematics (Inria) and is free of charge through a web-based user interface (http://www-tapenade. inria.fr:8080/tapenade/).

\section{Results}

The source transformation of the computer program was done using the multi-directional tangent (i.e. forward) mode of TAPENADE. The multi-directional mode allows for efficient execution of the program because redundant executions of primal operations are avoided. The source-transformed computer program was thus used to construct full Jacobian matrices and took just 4.5 times longer to run than the original program. Since the Jacobian matrices were not sparse, optimisation based on sparsity was not motivated.

In this work, if an input variable to the model was solely used in a lookup table, that input was replaced by the parameter that results from the lookup table (Appendix B). Namely, precipitation and state-of-the-ground input data are used in a lookup table to estimate a value for the Priestley-Taylor moisture parameter $\alpha$, whereas state of the ground is used to estimate the surface albedo $(r)$. From a sensitivity study point of view, it makes more sense to be able to assess the sensitivity to $\alpha$ and $r$ directly, rather than the sensitivity involving the lookup table procedure. Therefore, in this work, the lookup table variables $r$ and $\alpha$ are included as inputs to the MPP-FMI, which also reduces the number of input variables to be analysed. Thus, the sensitivity analysis becomes more straightforward to interpret because inherent step functions of lookup tables are circumvented. 
In addition to replacing the lookup table with parameters that result from the lookups, the sunshine fraction has been replaced with net incoming solar radiation at the surface $\left(R_{\mathrm{S}}\right)$. Replacing the sunshine fraction with $R_{\mathrm{S}}$ is motivated by an increased availability of direct measurements of $R_{\mathrm{S}}$. Originally, the sunshine fraction is used in a regression to derive $R_{\mathrm{S}}$ (Karppinen et al., 1997).

\subsection{Obukhov length sensitivity}

We have selected the ranges of the input parameters for the sensitivity analysis to be the commonly occurring ones in the meteorological and environmental conditions in the city of Helsinki, Finland. For instance, the ambient temperatures were assumed to range from -20 to $+30^{\circ} \mathrm{C}$. These ranges have been presented in Table 1.

The values in Table 1 were then used to construct the Jacobian (Eq. 8) for every combination of the meteorological input variables. The rows of interest for this work are those rows in the Jacobian containing the sensitivity information of $L^{-1}$ and $u_{*}$ since they are needed in Gaussian dispersion models such as CAR-FMI and UDM-FMI to model turbulent dispersion. In addition to $L^{-1}$ and $u_{*}$, the Jacobian comprises sensitivity information for the quantities $Q_{\mathrm{H}}, Q_{\mathrm{E}}, Q^{*}$, and $\theta_{*}$ to the respective input variables listed in Table 1 .

The range and units of the input variables vary greatly. Therefore, the intercomparison of partial derivatives of the outputs with respect to the input data as such is not desirable. In order to make the partial derivatives intercomparable, the partial derivatives have been normalised by $10 \%$ of the input range of the respective input variables denoted $\Delta x_{i}$. The range of the input data is listed in Table 1. In Fig. 2, the sensitivity of the inverse Obukhov length $\left(L^{-1}\right)$ is shown for all combinations of the input parameters listed in Table $1 . L^{-1}$ describes the atmospheric stability; for neutral conditions, $L^{-1} \approx 0$. When $L^{-1} \ll 0$, the atmosphere is unstable, and when $L^{-1} \gg 0$, the atmosphere is stable. For clarity, Fig. 2 is further separated into a low wind-speed situation with all other input variables varied (the main figure). The insert figure contains all combinations of input parameters associated with wind speeds in the range of $4-20 \mathrm{~m} \mathrm{~s}^{-1}$. The figure is separated into low and high wind-speed situations because the model is much more sensitive to input data when the wind speed is low; $U \approx 1 \mathrm{~ms}^{-1}$.

An obvious conclusion based on the results in Fig. 2 is that the wind speed $U$ is the most important parameter, and the solar irradiation $R_{\mathrm{S}}$ is the second most important one, with respect to the predicted values of the inverse Obukhov length. This result could also be physically expected, since wind speed is the most obvious factor in terms of the formation of mechanical turbulence, whereas solar irradiation is a crucial parameter for the thermally induced turbulence. As can be seen from Fig. 2, $L^{-1}$ is most sensitive to a change in $U$. When compared to the insert $\left(4 \mathrm{~m} \mathrm{~s}^{-1} \leq U \leq 20 \mathrm{~m} \mathrm{~s}^{-1}\right)$, the sensitivity to a change in wind speed is more pronounced

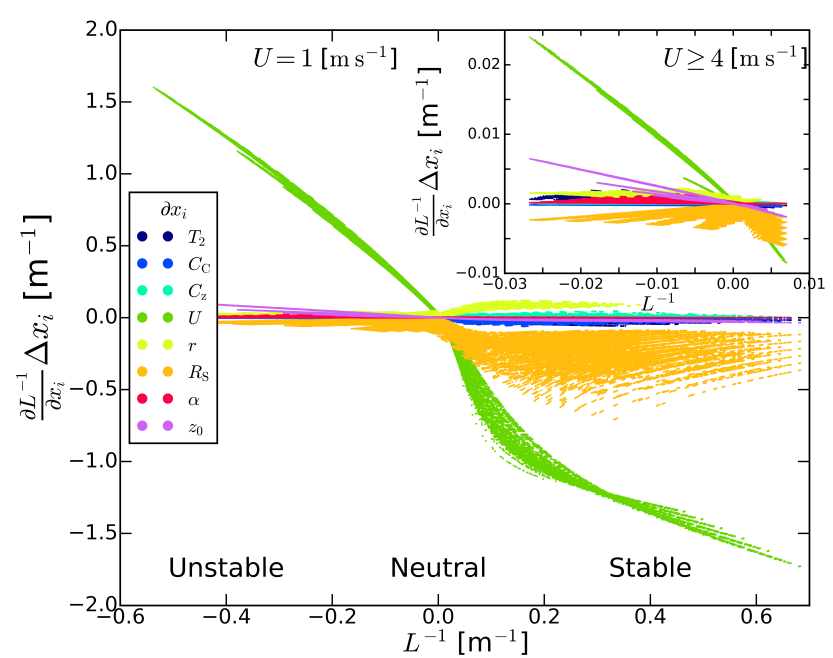

Figure 2. Sensitivity of inverse Obukhov length $\left(L^{-1}\right)$ with respect to input variables of MPP-FMI. The main figure shows sensitivities to all the input variables when the wind speed $(U)$ is $1 \mathrm{~m} \mathrm{~s}^{-1}$. The insert shows sensitivities for wind speeds in the range of 4$20 \mathrm{~m} \mathrm{~s}^{-1}$. In the figure, the partial derivatives have been normalised by the range of the input parameters $\left(\Delta x_{i}\right)$ described in Table 1 in order to make them intercomparable.

at low wind speeds. When $L^{-1}$ is negative, which is the case of unstable and neutral conditions, the partial derivative $\partial L^{-1} / \partial U$ is positive. That means that an increase in $U$ will always favour the modelled stability to become more neutral. That is, a negative $L^{-1}$ and a positive partial derivative of $\partial L^{-1} / \partial U$ will tend to move $L^{-1}$ towards neutral given that $U$ increases. Conversely, when $L^{-1}>0$ (i.e. stable to neutral), then $\partial L^{-1} / \partial U$ is always negative. This means that an increase in $U$ will therefore, again, tend to make $L^{-1}$ move towards neutral. This is in agreement with what one would expect in nature since an increase in $U$ will induce mechanical turbulence regardless of the initial stability and hence favour neutral conditions. At higher values of $U$, seen in the insert of Fig. 2, the $L^{-1}$ range is now restricted to roughly the range of $-0.03-0.01 \mathrm{~m}^{-1}$ (i.e. neutral).

The second most important input variable for the preprocessor with regard to $L^{-1}$ is $R_{\mathrm{S}}$. The partial derivative $\partial L^{-1} / \partial R_{\mathrm{S}}$ for all considered combinations of input values remains exclusively negative and even more so when $L^{-1}>0$. This means that an increase in $R_{\mathrm{S}}$ will always move the stability towards unstable. This follows the intuition that an increase in $R_{\mathrm{S}}$ will increase buoyancy-induced turbulence, therefore favouring an unstable boundary layer. At low wind speeds, it has to be noted that the spread in the sensitivity of $L^{-1}$ to $R_{\mathrm{S}}$ is an indication that other meteorological input variables influence the results, especially when $L^{-1}>0$. This is evident from the fact that the sensitivity to $R_{\mathrm{S}}$ does not follow a single line but is spread out. For example, when $L^{-1}=0.3 \mathrm{~m}^{-1}$, then $\partial L^{-1} / \partial R_{\mathrm{S}}$ is in the range of $-0.1-$ $0.6 \mathrm{~m}^{-1}$. The highest sensitivity to a change in $R_{\mathrm{S}}$, at low 
Table 1. Range of parameters used for studying the sensitivity of $L^{-1}$. For each range, six points were linearly spaced within the range. This amounts to $6^{8}$ (1.7 million) combinations of input variables to be evaluated, resulting in $6^{8}$ Jacobian matrices. In the table, $z_{0}$ is the roughness length, $r$ is the surface albedo, $T_{2}$ is the temperature at the height of $2 \mathrm{~m}, C_{\mathrm{C}}$ is the cloud cover, $U$ is the wind speed at $10 \mathrm{~m}, \alpha$ is the Priestley-Taylor moisture parameter, and $R_{\mathrm{S}}$ is the solar irradiance.

\begin{tabular}{rrrrrrrrr}
\hline Inputs & $z_{0}(\mathrm{~m})$ & $r$ & $T_{2}\left({ }^{\circ} \mathrm{C}\right)$ & $C_{\mathrm{C}}$ & $C_{\mathrm{Z}}(\mathrm{m})$ & $U\left(\mathrm{~m} \mathrm{~s}^{-1}\right)$ & $\alpha$ & $R_{\mathrm{S}}\left(\mathrm{W} \mathrm{m}^{-2}\right)$ \\
\hline Range & $0.3-1.3$ & $0.05-0.7$ & $-20-30$ & $0-1$ & $30-6000$ & $1-20$ & $0.5-1.0$ & $0-900$ \\
\hline
\end{tabular}

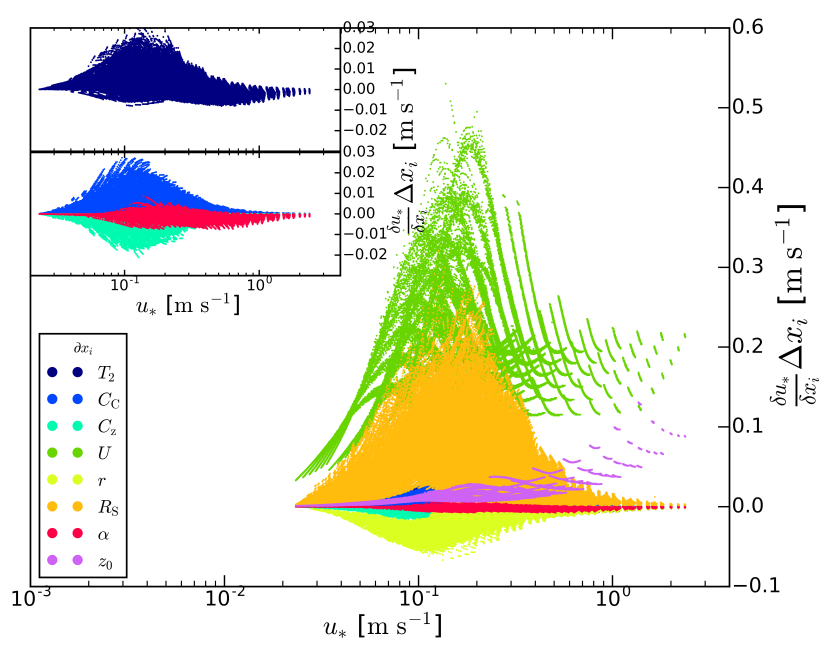

Figure 3. Sensitivity of friction velocity $\left(u_{*}\right)$ with respect to input variables of MPP-FMI. The main figure shows sensitivities of the most important input variables, whereas the inserts show the less sensitive input variables. The partial derivatives have been normalised by the range of the input parameters $\left(\Delta x_{i}\right)$ described in Table 2 in order to make them intercomparable.

wind speeds, is when $R_{\mathrm{S}}$ is close to zero and the surface albedo $(r)$ is low. This information is, however, not colour coded into the figure (so as not to degenerate the clarity of the figure).

\subsection{Friction velocity sensitivity}

Another important scaling parameter for Gaussian models is $u_{*}$. Moreover, $u_{*}$ is also central for the iteration procedure in the preprocessor when finding a value for $L^{-1}$. Table 2 summarises the input variable ranges for the $u_{*}$ sensitivity analysis. The variable range used for the sensitivity study of $u_{*}$ differs from that of $L^{-1}$ in the case of the selected wind speeds; the extremely high wind speeds (from 12 to $20 \mathrm{~m} \mathrm{~s}^{-1}$ ) have been omitted in the case of the $u_{*}$ sensitivity analysis. The latter selection was made in order to be able to present the results more clearly; the highest wind speeds also occur only for a small fraction of time. The sensitivity of $u_{*}$ to different input variables is depicted in Fig. 3.

As for the corresponding results for $L^{-1}$, the wind speed $U$ was the most important parameter, and the solar irradiation $R_{\mathrm{S}}$ was the second most important one. This result is physically to be expected also in the case of the sensitivity of $u_{*}$.

Amongst the input parameters, only $U$ and $z_{0}$ are present in the equation for $u_{*}$. The rest of the sensitivity of $u_{*}$ is, to a varying degree, related to the cross sensitivity between $L^{-1}$ and $u_{*}$ through Eqs. (2)-(5). Since $u_{*}$ is a scaling parameter for the production of turbulent kinetic energy due to shear stress, $u_{*}$ is generally high for high values of $U$. Thus, a generalisation can be made that $u_{*}$ is most sensitive to $U$ at low wind speeds. Furthermore, the stability functions $\psi_{\mathrm{M}}$ of Eq. (2) will increase $u_{*}$ the more negative (unstable) $L^{-1}$ becomes and decrease $u_{*}$ the more positive (stable) $L^{-1}$ becomes; see Appendix A. For neutral stability $\left(L^{-1} \approx 0\right)$, the stability functions $\psi_{\mathrm{M}}$ of Eq. (2) yield very similar results for $u_{*}$. At higher wind speeds, the value of $z_{0}$ determines to a greater extent the sensitivity of $\partial u_{*} / \partial U$. This is clearly visible when $u_{*}>1 \mathrm{~m} \mathrm{~s}^{-1}$ as six vertically separated groups of points in Fig. 3; six groups are used because of six different values of $z_{0}$. This is, however, not colour coded into the figure so as not to degenerate the clarity of the figure.

The second most important input parameter for $u_{*}$ is $R_{\mathrm{S}}$. This holds true for low values of $u_{*}$. Based on the discussion regarding the sensitivity of $L^{-1}$, this is expected. However, from Eq. (2), it is not that clear that $u_{*}$ is sensitive to the solar radiation input into the preprocessor. Again, as $R_{\mathrm{S}}$ changes, this will impact the absolute values that comprise the energy budget equation; see Eq. (1). This in turn will impact $\theta_{*}$ which consequently impacts $L^{-1}$ and ultimately $u_{*}$ through the stability functions. However, at high $u_{*}$, the importance of $z_{0}$ will be more important for the modelled value of $u_{*}$ than $R_{\mathrm{S}}$ as depicted in the figure. Opposite to the sensitivities to $U, R_{\mathrm{S}}$, and $z_{0}$, an increase in surface albedo $(r)$ will lower $u_{*}$ through $L^{-1}$.

\subsection{Cross sensitivity}

The sensitivity study of $L^{-1}$ and $u_{*}$ has shown that $U$ is the most important parameter for MPP-FMI. $L^{-1}$ is highly sensitive to a change in $U$ when $U \approx 1 \mathrm{~m} \mathrm{~s}^{-1}$. Moreover, $u_{*}$ is also most sensitive to $U$. Because $u_{*}$ is a function of $L^{-1}$ (Eq. 2) and $L^{-1}$ is a function of $u_{*}$ (Eq. 4), these scaling parameters are interconnected. Thus, these scaling parameters are cross sensitive.

Figure 4 shows the cross sensitivity between $\partial u_{*} / \partial U$ and $L^{-1}$. The figure shows that the largest sensitivity of $\partial u_{*} / \partial U$ 
Table 2. Range of parameters used for studying the sensitivity of $u_{*}$. Six points were linearly spaced within the range, except for $U$ which comprises 10 logarithmically spaced points which amount to roughly 2.8 million combinations of input variables. In the table, $z_{0}$ is the roughness length, $r$ is the surface albedo, $T_{2}$ is the temperature at the height of $2 \mathrm{~m}, C_{\mathrm{C}}$ is the cloud cover, $U$ is the wind speed at $10 \mathrm{~m}, \alpha$ is the Priestley-Taylor moisture parameter, and $R_{\mathrm{S}}$ is the solar irradiance.

\begin{tabular}{rrrrrrrrr}
\hline Inputs & $z_{0}(\mathrm{~m})$ & $r$ & $T_{2}\left({ }^{\circ} \mathrm{C}\right)$ & $C_{\mathrm{C}}$ & $C_{\mathrm{Z}}(\mathrm{m})$ & $U\left(\mathrm{~m} \mathrm{~s}^{-1}\right)$ & $\alpha$ & $R_{\mathrm{S}}\left(\mathrm{W} \mathrm{m}^{-2}\right)$ \\
\hline Range & $0.3-1.3$ & $0.05-0.7$ & $-20-30$ & $0-1$ & $30-6000$ & $1-12$ & $0.5-1.0$ & $0-900$ \\
\hline
\end{tabular}

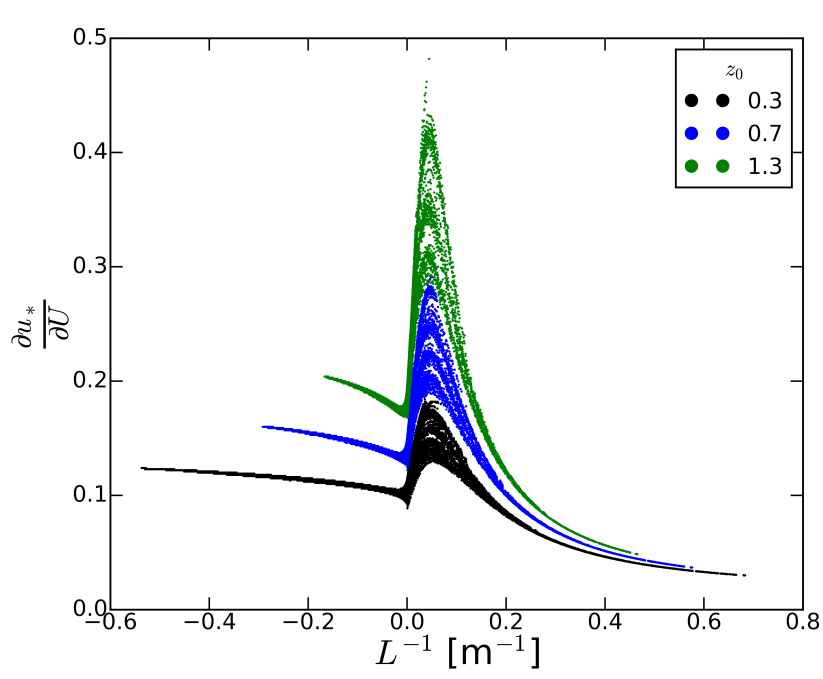

Figure 4. Cross sensitivity between atmospheric stability $\left(L^{-1}\right)$ and friction velocity $\left(u_{*}\right)$ with respect to wind speed $(U)$ for different surface roughness lengths $\left(z_{0}\right)$. Not all $z_{0}$ values in the range are plotted for clarity. Note that the $y$ axis of Fig. 4 is not scaled as in the previous figures because there is no intercomparison between different input data in this figure.

will be when $L^{-1}$ is around $0.1 \mathrm{~m}^{-1}$, i.e. mildly stable. The different behaviour of $\partial u_{*} / \partial U$ when $L^{-1}>0$ is likely due to the increased complexity of the stability functions $\left(\psi_{\mathrm{M}}\right.$ in Eq. 2) for stable conditions than for unstable conditions; see Appendix A for details. This behaviour is not captured in Figs. 2 and 3 although it could perhaps be inferred. This behaviour is also likely to be the case for real atmospheric conditions since a mildly stable boundary layer would be susceptible to increasing $U$ and consequently the production of wind-shear-induced turbulence which would cause $u_{*}$ to increase. For highly stable conditions, the sensitivity of $\partial u_{*} / \partial U$ levels out and is below the sensitivity for unstable conditions.

For unstable conditions $\left(L^{-1} \ll 0\right)$, the sensitivity of $\partial u_{*} / \partial U$ is less complex and the degree of sensitivity is largely dictated by $z_{0}$, which also holds true for mildly stable conditions. Without the stability functions $\psi_{\mathrm{M}}$ and $\psi_{\mathrm{H}}$, a cross sensitivity would still remain; however, it is not as intricate as depicted in Fig. 4.

\section{Conclusions and discussion}

The sensitivities of the meteorological preprocessor model MPP-FMI on its input values were examined by the means of algorithmic differentiation. The differentiation of the preprocessor was carried out by a source-transformation AD tool called TAPENADE, yielding a program that evaluates the desired sensitivity derivatives with machine precision. We focused on the evaluation of vertical fluxes in the atmosphere and in particular on the sensitivity of the predicted inverse Obukhov length and friction velocity on the model input parameters. These two quantities were selected as they are key parameters in view of air pollution.

The study shows that the predicted inverse Obukhov length and friction velocity are most sensitive to wind speed and, second most importantly, to solar irradiation. The dependency on wind speed is most pronounced at low wind speeds. For both predicted inverse Obukhov length and friction velocity, the third most important factors are the roughness length and the surface albedo, for unstable and stable conditions, respectively. The surface roughness length determines how sensitive the friction velocity is to wind speed.

The presented results have implications for improving the meteorological preprocessing models and for selecting and preparing the measured input values for such models. For instance, the high sensitivity of the preprocessor to the values of the wind speed at the height of $10 \mathrm{~m}$ implies that the wind observations have to be selected very carefully. Clearly, the wind-speed observations should be as representative as possible for the whole of the domain to be considered and should not be affected or substantially influenced by any local disturbances.

Finally, another key parameter worthy of study for atmospheric dispersion models is mixing height, because the mixing height describes the depth of lowermost layer in which pollutants disperse.

This study gave more confidence that $\mathrm{AD}$, in general, and the TAPENADE tool in particular are useful tools of assessment for studying quantitatively the ranges of sensitivities of the predicted parameters. The analysis is more comprehensive and versatile compared with the use of previously applied sensitivity analysis methods. The sensitivities could be analysed for a wide range of input conditions both accurately and effectively. 
The AD procedure is also useful for analysing the functioning of computer programs, and for improving their optimisation in terms of computing resources. In this study, all the dependencies of the predicted parameters on the model input values were found to be physically understandable and feasible. However, the procedure could also be useful for finding out potential inaccuracies of the numerical solutions, or even mistakes in the structure of the computer codes.

The meteorological preprocessor parameterisation scheme (that is originally based on van Ulden and Holtslag) used in this study is in fairly common use in other countries within meteorological preprocessors and dispersion models. The initial conditions used in the model computations corresponded to the climate and environmental conditions in Helsinki. However, the range of conditions at such a northern latitude vary substantially (for instance, the ambient temperatures were assumed to range from -20 to $+30^{\circ} \mathrm{C}$ ), and the more moderate climatic conditions that are common for most of central Europe are actually included in the selected wide variability. The main insights and conclusions found out in this study are therefore probably similar for several other preprocessors used in Europe that use the same or a similar boundary-layer scaling method.

Future research could address the determination of how the sensitivity of MPP-FMI impacts the modelled concentrations of pollutants. Such research could be done by source transforming a chain of models using AD, instead of only one model. The next chain of models to be investigated could be a combination of a meteorological preprocessor and an urban-scale dispersion model. The sensitivity of the combined modelling system could also be evaluated in terms of other input values of the dispersion model, in addition to the meteorological ones.

Code availability. The source code for the meteorological preprocessor (MPP-FMI 3.0) is included in the Supplement. The source-transformed code is also included in the Supplement. The source-transformed code is subject to the TAPENADE licence agreement which limits the use of the code to academic research (see www-sop.inria.fr/tropics/tapenade/downloading.html). The Supplement also contains the code that was used to produce the input data and a wrapper to handle data input and output. 


\section{Appendix A}

The empirical stability functions of Eq. (2) as implemented in the meteorological preprocessor are

$\psi_{\mathrm{M}}=(1-16 z / L)^{1 / 4}-1$ for $L<0$

$\psi_{\mathrm{M}}=-17\left(1-e^{-0.29 z / L}\right)$ for $L>0$.

The stability functions of Eq. (A1) are taken from Karppinen et al. (1997). Figure A1 shows $u_{*}$ as a function of $L^{-1}$ for two different wind speeds $\left(1\right.$ and $\left.4 \mathrm{~m} \mathrm{~s}^{-1}\right)$. Note that $-L^{-1}$ and $L^{-1}$ are plotted on the same $x$ axis.

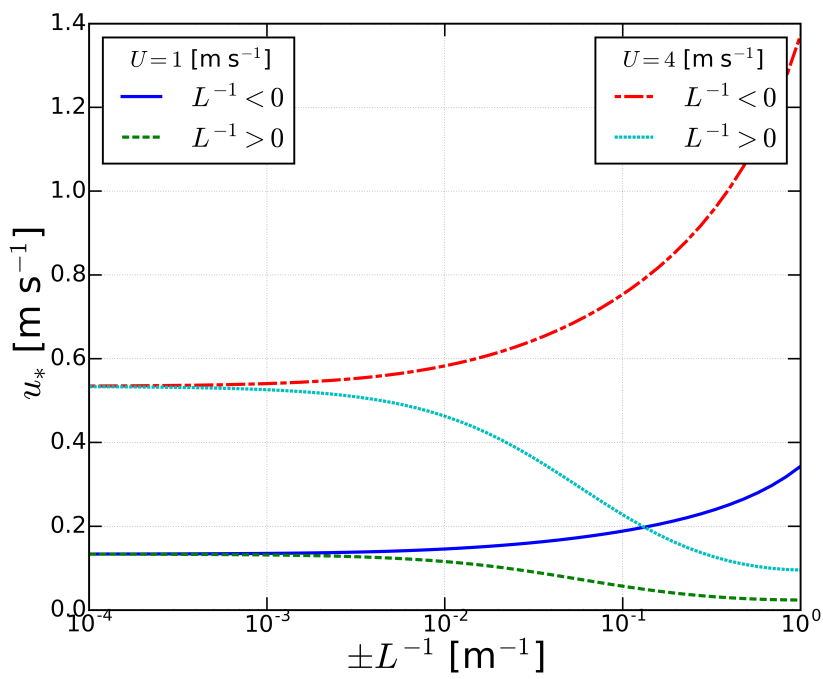

Figure A1. Friction velocity $\left(u_{*}\right)$ as a function of inverse Obukhov length $\left(L^{-1}\right)$ for two different wind speeds $(U)$ using a roughness length $\left(z_{0}\right)$ of $0.5 \mathrm{~m}$ and wind-speed measurement height $(z)$ of $10 \mathrm{~m}$.

\section{Appendix B}

This appendix covers the lookup table parameters that are used to estimate the surface albedo $(r)$ and the PriestleyTaylor moisture parameter $(\alpha)$.

The state of the ground is used in a lookup table to obtain an estimate for the surface albedo according to surface type and the state of the ground. The lookup table procedure is shown in Table B1.

The Priestley-Taylor parameter estimate is estimated using a lookup table involving weather codes, solar elevation angle, state of the ground, and precipitation during the last $12 \mathrm{~h}$ (Karppinen et al., 1997). The lookup table is illustrated by a flow chart in Fig. B1.

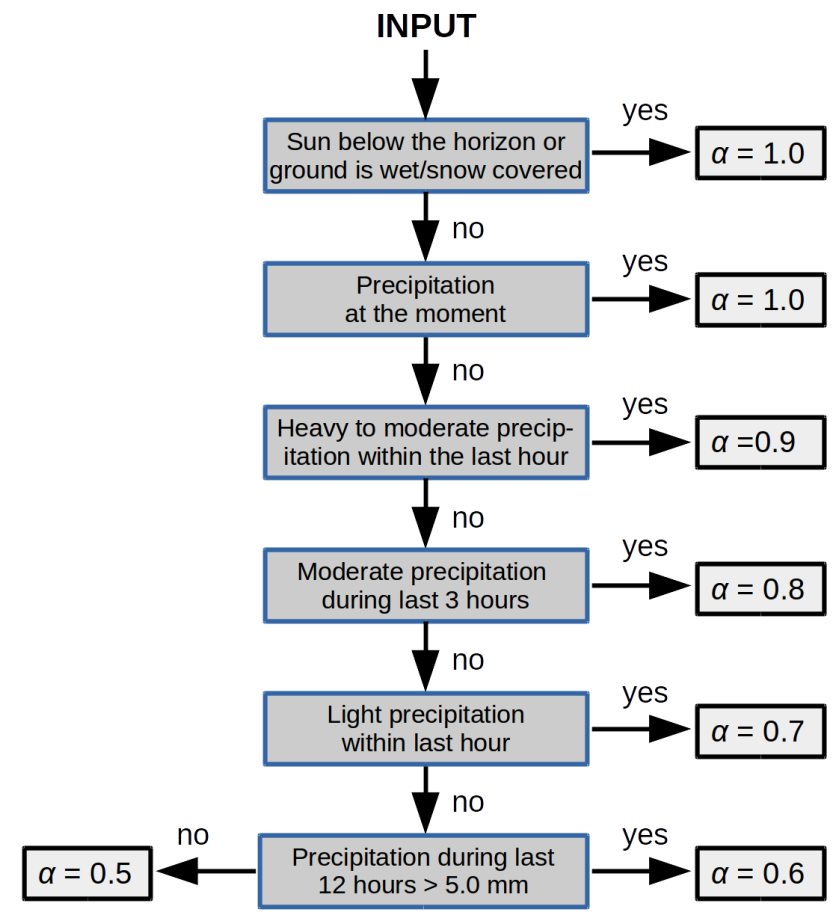

Figure B1. Flow chart of how the Priestley-Taylor moisture parameter $(\alpha)$ is estimated from input parameters that comprise state of the ground, current weather, weather during the last hour, weather during the last $3 \mathrm{~h}$, precipitation during last $12 \mathrm{~h}$, and solar elevation angle. 
Table B1. Lookup table for surface albedo $(r)$ based on surface type and state of the ground.

\begin{tabular}{|c|c|c|c|c|c|c|c|c|c|c|}
\hline & \multicolumn{10}{|c|}{ State of the ground } \\
\hline & \multicolumn{3}{|c|}{ Soil } & \multicolumn{2}{|c|}{ Ice } & \multicolumn{5}{|c|}{ Snow cover $(\%)$} \\
\hline & Dry & Moist & Wet & Dry & Wet & $<50$ & $50<100$ & 100 & $50<100$ & 100 \\
\hline Surface & & & & & & Melting & Melting & Melting & Dry snow & Dry snow \\
\hline Sea & 0.06 & 0.06 & 0.06 & 0.06 & 0.06 & 0.30 & 0.30 & 0.70 & 0.71 & 0.71 \\
\hline Lake & 0.05 & 0.05 & 0.05 & 0.15 & 0.15 & 0.18 & 0.38 & 0.71 & 0.71 & 0.71 \\
\hline Wasteland & 0.13 & 0.13 & 0.13 & 0.13 & 0.33 & 0.44 & 0.55 & 0.67 & 0.67 & 0.67 \\
\hline Field & 0.2 & 0.2 & 0.2 & 0.13 & 0.11 & 0.33 & 0.55 & 0.67 & 0.67 & 0.67 \\
\hline Forest & 0.11 & 0.11 & 0.11 & 0.11 & 0.17 & 0.26 & 0.34 & 0.39 & 0.39 & 0.39 \\
\hline City & 0.22 & 0.22 & 0.22 & 0.13 & 0.11 & 0.17 & 0.22 & 0.28 & 0.28 & 0.39 \\
\hline
\end{tabular}




\section{The Supplement related to this article is available online at https://doi.org/10.5194/gmd-10-3793-2017- supplement.}

Competing interests. The authors declare that they have no conflict of interest.

Acknowledgements. This work was funded by the Maj and Tor Nessling Foundation (grants 2014044, 201600449, and 201700305).

Edited by: David Ham

Reviewed by: Laurent Hascoet and one anonymous referee

\section{References}

Fisher, B., Kukkonen, J., and Schatzmann, M.: Meteorology applied to urban air pollution problems COST 715, Int. J. Environ. Pollut., 16, 560-570, https://doi.org/10.1504/IJEP.2001.000650, 2001.

Griewank, A. and Walther, A.: Evaluating Derivatives Principles and Techniques of Algorithmic Differentiation, vol. 2, Society for Industrial and Applied Mathematics, Philadelphia, USA, 1$56,2008$.

Guerrette, J. J. and Henze, D. K.: Development and application of the WRFPLUS-Chem online chemistry adjoint and WRFDAChem assimilation system, Geosci. Model Dev., 8, 1857-1876, https://doi.org/10.5194/gmd-8-1857-2015, 2015.

Hascoet, L. and Pascual, V.: The Tapenade Automatic Differentiation Tool: principles, model, and specification, ACM T. Math. Software, 39, 20:1-20:43, https://doi.org/10.1145/2450153.2450158, 2013.

Karppinen, A., Joffre, S. M., and Vaajama, P.: Boundary-layer parameterization for Finnish regulatory dispersion models, Int. J. Environ. Pollut., 8, 3-6, 1997.

Karppinen, A., Joffre, S. M., and Kukkonen, J.: The refinement of a meteorological preprocessor for the urban environment, Int. J. Environ. Pollut., 14, 1-9, https://doi.org/10.1504/IJEP.2000.000580, 2000a.

Karppinen, A., Kukkonen, J., Elolähde, T., Konttinen, M., Koskentalo, T., and Rantakrans, E.: A modelling system for predicting urban air pollution: model description and applications in the Helsinki metropolitan area, Atmos. Environ., 34, 3723-3733, https://doi.org/10.1016/S1352-2310(00)00074-1, 2000 b.
Karppinen, A., Joffre, S. M., Kukkonen, J., and Bremer, P.: Evaluation of inversion strengths and mixing heights during extremely stable atmospheric stratification, Int. J. Environ. Pollut., 16, 1-6, https://doi.org/10.1504/IJEP.2001.000653, 2001.

Kauhaniemi, M., Karppinen, A., Härkönen, J., Kousa, A., Alaviippola, B., Koskentalo, T., Aarnio, P., Elolähde, T., and Kukkonen, J.: Evaluation of a modelling system for predicting the concentrations of $\mathrm{PM}_{2.5}$ in an urban area, Atmos. Environ., 42, 4517-4529, https://doi.org/10.1016/j.atmosenv.2008.01.071, 2008.

Kim, J. G., Hunke, E. C., and Lipscomb, W. H.: Sensitivity analysis and parameter tuning scheme for global sea-ice modeling, Ocean Model., 14, 61-80, https://doi.org/10.1016/j.ocemod.2006.03.003, 2006.

Kukkonen, J., Härkönen, J., Walden, J., Karppinen, A., and Lusa, K.: Validation of the dispersion model CAR-FMI against measurements near a major road, Atmos. Environ., 35, 949-960, https://doi.org/10.1016/S1352-2310(00)00337-X, 2001.

Schreier, F., Gimeno García, S., Hedelt, P., Hess, M., Mendrok, J., Vasquez, M., and $\mathrm{Xu}$, J.: GARLIC - a general purpose atmospheric radiative transfer line-by-line infrared-microwave code: implementation and evaluation, J. Quant. Spectrosc. Ra., 137, 29-50, https://doi.org/10.1016/j.jqsrt.2013.11.018, 2014.

Schreier, F., Gimeno García, S., Vasquez, M., and Xu, J.: Algorithmic vs. finite difference Jacobians for infrared atmospheric radiative transfer, J. Quant. Spectrosc. Ra., 164, 147-160, https://doi.org/10.1016/j.jqsrt.2015.06.002, 2015.

Van Ulden, A. P. and Holtslag, A. A. M.: Estimation of atmospheric boundary layer parameters for diffusion applications, J. Clim. Appl. Meteorol., 24, 1196-1207, https://doi.org/10.1175/15200450(1985)024<1196:EOABLP>2.0.CO;2, 1985.

Wood, C. R., Järvi, L., Kouznetsov, R. D., Nordbo, A., Joffre, S., Drebs, A., Vihma, T., Hirsikko, A., Suomi, I., Fortelius, C., O’Connor, E., Moiseev, D., Haapanala, S., Moilanen, J., Kangas, M., Karppinen, A., Vesala, T., and Kukkonen, J.: An overview of the urban boundary layer atmosphere network in Helsinki, B. Am. Meteorol. Soc., 94, 1675-1690, https://doi.org/10.1175/BAMS-D-12-00146.1, 2013. 